\title{
LOGISTIK Innovations- und Kompetenz-Zentrum TGZ Wildau - LoWA-TGZ
}

\author{
Logistik - Weiterbildung - Ansiedlung \\ Gedanken zur Profilbildung des Technologie- und Gründerzentrums Wildau
}

\author{
Eberhard Brink, Bernd Hentschel, Hartmut Stoltenberg
}

Das Technologie- und Gründerzentrum Wildau tritt in wenigen Monaten in die entscheidende Phase seiner Entwicklung ein. Anfang des Jahres 2000 werden die ersten Firmen in die sanierten, denkmalgeschuitzen Konstruktionsgebäude des früheren VEB Schwermaschinenbau „Heinrich Rau“ - vormals Fa. SchwartzkopffMaffei (Abb. 1) - einziehen. Ein zweigeschossiger Neubau, in dem auch größere Maschinen und Anlagen aufgestellt werden können, ergänzt dann die Altgebäude (Abb. 2). Mit der Eröffnung des TGZ - eigenen Gebäudekomplexes in der Freiheitstr. 124/126 sind die Voraussetzungen gegeben, in den nächsten drei bis vier Jahren bis zu 50 Unternehmen mit 200 Arbeitsplätzen in Wildau anzusiedeln, Synergieeffekte zu organisieren und die Zusammenarbeit mit der Technischen Fachhochschule Wildau (TFH) weiter auszubauen.

In Deutschland gibt es mehr als 250 Technologie- und Gründerzentren, im Land Brandenburg 21 und in Berlin mit seinem engeren Verflechtungsraum allein 15; Tendenz steigend!

Wie kann, wie wird sich zukünftig das Wildauer Zentrum in diesem Ensemble darstellen?

Mit den geplanten $4.000 \mathrm{~m}^{2}$ Mietfläche wird das TGZ Wildau eher zu den kleineren Zentren gehören. Dadurch werden intensivere Kontakte der Mieter untereinander, mit der benachbarten Hochschule und mit dem Zentrumsmanagement begünstigt.

Die Standortvorteile Wildaus wurden bereits in früheren Beiträgen ausführlich dargestellt (1).

Was macht darüberhinaus die Chancen eines Technologiezentrums aus?

G. Kayser vom Institut für Mittelstandsforschung in Bonn führte dazu in einem Interview aus: „Chancen haben aus meiner Sicht nur hochtechnologische Gründerzentren, die ganz klar umrissene Standorteigenschaften aufweisen muissen. Hierzu zählen die Vernetzung mit einer angegliederten Uni oder Forschungseinrichtung, Kommunikationskanäle zu Finanziers, Vermarktungseinrichtungen, hinreichend ausgebildetes Humankapital, das auch bereit ist, in einer Region zu bleiben, die also auch durch Freizeitwerte attraktiv ist, etc. etc." (2).
Erfolgreiche Technologiezentren zeichnen sich durch Spezialisierung und Vernetzung aus. Beispiele im Land Brandenburg hierfür sind der Biotechnologiepark Luckenwalde und das Technologiezentrum für Verkehrstechnik in Hennigsdorf.

Auf der Grundlage der Gegebenheiten des Standortes Wildau gilt es, erfolgversprechende Spezialisierungsmöglichkeiten und geeignete Partner zu finden. Je besser es gelingt, einen attraktiven Technologieschwerpunkt glaubhaft zu entwickeln, umso eher wird das Zentrum Kompetenz auf diesem Gebiet erwerben und eigenes Profil herausbilden. Dabei sollten Felder belegt werden, die eine überproportionale Entwicklung erwarten lassen und in Berlin und Brandenburg in dieser Weise noch nicht vertreten sind.

Im Herbst 1998 hatte das Ministerium für Wirtschaft, Mittelstand und Technologie des Landes Brandenburg einen Regionalwettbewerb ausgelobt, in dem die brandenburgischen Technologiezentren aufgefordert wurden, neue Initiativen zur Profilierung ihrer Einrichtung, zur regionalen Vernetzung, zu Existenzgründungs- und Ansiedlungsaktivitäten zu entwickeln. In dieser Skizze werden Überlegungen weitergeführt, die vom TGZ Wildau in Zusammenarbeit mit der TFH Wildau als Beitrag eingereicht worden waren (3).

Die für das TGZ Wildau angestrebte Spezialisierung sollte auf dem Gebiet der Logistik liegen, mit der gesamten Komplexität, die diese Fachdisziplin von der Fabrikplanung und Produktionslogistik bis zur Telematik und Verkehrstechnik zu bieten hat.

Unter Nutzung des entscheidenden Wildauer Standortvorteils, der engen Zusammenarbeit mit der Technischen Fachhochschule Wildau, und in Vernetzung mit leistungsfähigen Unternehmen der Region stellt sich das Technologie- und Gründerzentrum Wildau das Ziel, das Profil für das

$$
\begin{gathered}
\text { LOGISTIK Innovations- und Kompetenz- } \\
\text { Zentrum - TGZ Wildau } \\
\text { „LoWA - TGZ-Wildau“ } \\
\text { als Einheit von } \\
\text { Logistik - Weiterbildung - Ansiedlung }
\end{gathered}
$$

zu erarbeiten. 


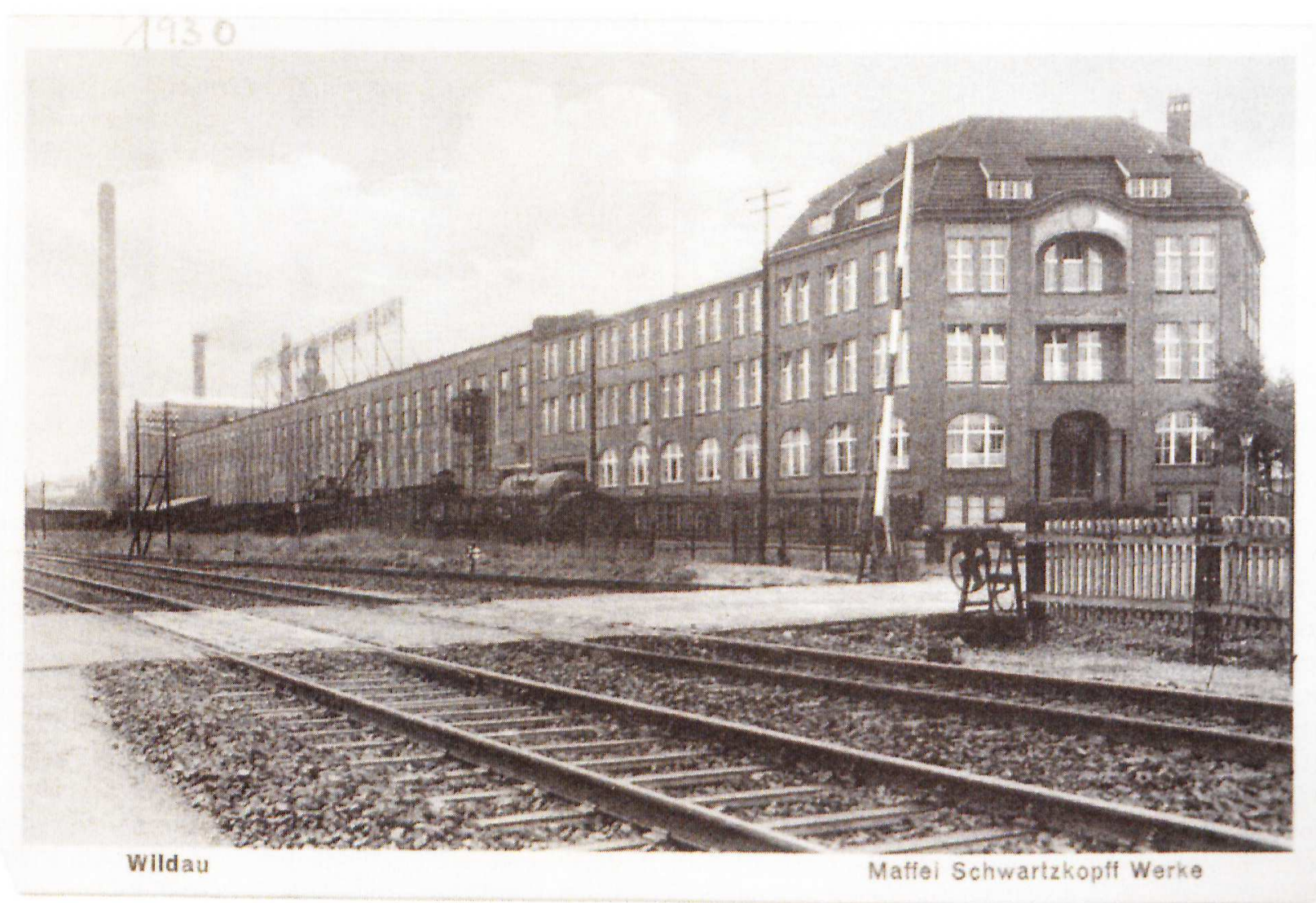

Abb. 1

Der Kurzname knuipft an die aus der Nachkriegszeit gebräuchliche Bezeichnung LOWA-Gebäude für die vom TGZ erworbenen denkmalgeschuitzten Altgebäude an. Von 1949 bis 1953 waren diese von der Vereinigung Volkseigener Betriebe Lok- und Waggonbau (VVB LOWA) als zentrales Verwaltungsgebäude genutzt worden.

Im „Logistik-Innovations- und Kompetenzzentrum - TGZ Wildau“ sollen ausgerichtet auf das fachliche Profil Logistik innovationsfördernde Kooperationen, fachspezifische Weiterbildung und die konforme Ansiedlung junger Firmen zu einer Einheit zusammengefuihrt werden.

Mit der Profilierung der Technischen Fachhochschule Wildau zum Logistikkompetenzzentrum fuir Osteuropa wird parallel iiber das Technologie- und Gründerzentrum Wildau (TGZ) ein unternehmensübergreifendes Innovations- und Kompetenzzentrum als fachliche Ergänzung errichtet und durch innovative Unternehmensansiedlungen komplettiert. Damit wird die enge Verflechtung von logistischer Ausbildung mit konkreter Projektarbeit im nationalen und internationalen Rahmen gesichert. Die Profilierung und Ausrichtung dieses Logistik-Innovations- und Kompetenz-Zentrums im TGZ soll sich insbesondere auf folgende inhaltliche Schwerpunkte in enger Verbindung mit Technischen Fachhochschule Wildau ausrichten:

1. Virtuelle Prozeß- und Logistiksysteme

2. Prozeß- und Logistikinnovationen für in- und ausländische Unternehmen

3. Innovative Entsorgungslogistiksysteme

4. Qualitätsmanagementsysteme in Logistikprozessen
Damit könnten attraktive Leistungsinhalte aus den Bereichen der Logistik von Unternehmen aus Wildau angeboten werden. Zusammen mit den dazu erforderlichen Logistikdienstleistungen für die Region BerlinBrandenburg und den osteuropäischen Raum würden sich diese zu einem erheblichen wirtschaftlichen Faktor ausweiten.

Mehrere Unternehmen haben ihr Ansiedlungsinteresse nachhaltig bekundet. Darunter befinden sich auch solche, die bereits iiber Erfahrungen auf diesen neuen Märkten verfuigen und eine Leitfunktion übernehmen könnten. Dazu gehört u.a. die Firma Projektlogistik $\mathrm{GmbH}$, die als Logistikdienstleister des Berlin-Brandenburger Wirtschaftsraumes mit Ausstrahlung nach Polen und den benachbarten Bundesländern bereits eine Vielzahl von Projekten mit renommierten Unternehmen bearbeitet hat und somit zu einer festen Größe im Logistik-Innovations- und Kompetenz-Zentrum Wildau werden wird. Diese Firma verlagert ihren Sitz von Berlin vollständig nach Wildau. Zur Zeit ist sie schon mit einer Außenstelle in Wildau vertreten.

Zum anderen wird parallel zur unternehmensspezifischen Projektarbeit die internationale Kooperation ausgebaut. Die von der Hochschule ausgerichtete Deutsch-Polnische Logistikkonferenz gehört schon nach wenigen Jahren - im Mai 1999 fand in Slubice / Frankfurt/O. - die Vierte in Folge statt (4) - zum untrennbaren Bestandteil der Logistikaktivitäten aus Wildau. Zukünftig werden diese Konferenzen unter aktiver Einbeziehung des TGZ Wildau mit den dort verbundenen Unternehmen zu organisieren sein. 
Die Kontakte der TFH Wildau reichen im Rahmen der internationalen Logistikausbildung nicht nur nach Poznan im Nachbarland Polen und in andere europäische Länder, sondern seit kurzem sogar bis nach Brasilien, zum dortigen Logistikinstitut IBRALOG. Durch konkrete Projektarbeit, die zusammen mit der Landesentwicklungsgesellschaft für Städtebau, Wohnen und Verkehr des Landes Brandenburg mbH durchgeführt werden soll, sind durch länderuibergreifende innovative Unternehmensgründungen und -ansiedlungen für das TGZ Wildau zu erwarten.

Von diesem Leistungsangebot könnten dann sogar renommierte Unternehmen der Region, wie der Flughafen Schönefeld, Rolls-Royce Dahlewitz, MTU Ludwigsfelde, Daimler-Chrysler Ludwigsfelde, Bombardier Transportation Berlin-Grünau, der Binnenhafen Königs Wusterhausen oder auch Cargo Lifter in Brand/Niederlausitz profitieren.

Somit wäre die strategische Ausrichtung des TGZ Wildau zusammen mit der TFH Wildau, verbunden mit Unternehmen der Region, auf die Schaffung eines leistungsstarken und innovativen Logistik-Kompetenz-Zentrums ausgerichtet, das die Möglichkeiten der Beteiligten in den globalen Märkten deutlich verbessern wird.

Um die Kontakte $\mathrm{zu}$ betreffenden Branchen der Wirtschaft zu intensivieren und an aktuellen Entwicklungen teilzuhaben, wurde das TGZ Wildau 1998 Mitglied der Berlin-Brandenburg Aerospace Allianz (BBAA) zur Förderung der Luftfahrt in der Region und - ebenfalls 1998 - zusammen mit der TFH Wildau Mitbegründer des Vereins zur Förderung der regionalen Verkehrsanbindung Königs Wusterhausen.

Die im TGZ Wildau ansiedelnden Firmen sollten vorzugsweise als Entwickler- und Dienstleister für Großunternehmen der Logistik und der Luftfahrt tätig werden. Dieses wirtschaftsstarke Umfeld macht einen Teil der Attraktivität des Standorts Wildau aus.

In einem grundständigen Studiengang werden seit 1997 an der TFH Wildau Fachleute auf dem Gebiet der Logistik ausgebildet (5). Die Hochschule versteht sich neben ihrem Bildungsauftrag als aktiver Partner für Unternehmen und Verwaltung. Hochschullehrer stehen als praxiserfahrene Consulting-Partner zur Verfügung. Studentische Praktika und Diplomarbeiten geben den Unternehmen qualifizierte Arbeitsleistung und Innovationsimpulse. Zugleich verbessern diese Kontakte erheblich die Arbeitsmarktchancen der Absolventen.

Das Rüstzeug für Existenzgründungen ist Teil der wissenschaftlichen Ausbildung. Darüberhinaus werden Existenzgründer-Seminare und Workshops zu aktuellen, wirtschaftsrelevanten Fragen angeboten.

Für die postgraduale Weiterbildung von Fach- und Führungskräften sind in Zusammenarbeit mit namhaften Unternehmen und dem TGZ unter Federführung der TFH Wildau die Logistik-Akademie Wildau und die Telematik-Akademie Wildau in Vorbereitung.

Partner des TGZ aus dem Umfeld der Wildauer Hochschule sind auch die Technologietransfer- und Inno-

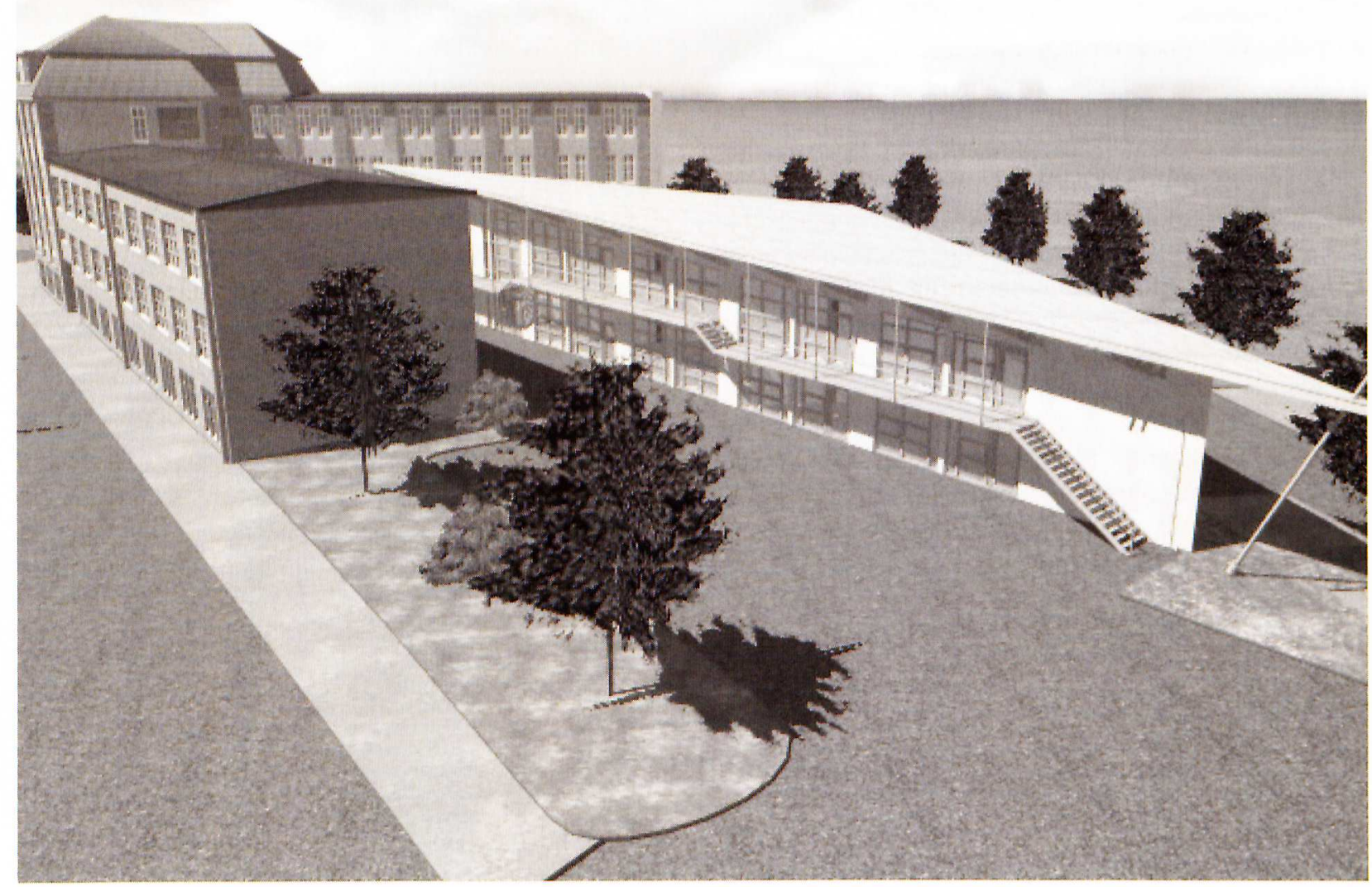


vationsberatungsstelle (TIB) und das Technologietransfer- und Weiterbildungszentrum (TWZ). Aufgabe der TIB ist vor allem die Intensivierung der Kontakte zwischen Wirtschaft und Hochschule. Das TWZ bietet Hochschullehrern geeignete Rahmenbedingungen für Institutsgrïdungen, die sich mit wirtschaftsnahen F\&E-Projekten beschäftigen. Derzeit sind im TWZ 15 Institute tätig, die auf sehr unterschiedlichen Gebieten Probleme von Wirtschaft und Verwaltung bearbeiten. Für die Profilbildung und Kompetenzentwicklung des TGZ sind insbesondere vom Institut für Unternehmenslogistik wichtige Impulse zu erwarten.

Die originäre Aufgabe eines TGZ ist die Ansiedlung von profilkonformen, leistungsfähigen Existenzgründern und Jungunternehmern. Dabei geht es nicht nur um die gute Auslastung der Mietflächen, sondern vor allem auch um Impulse für den Strukturwandel in der Region und um Beiträge zur Verbesserung ihrer Lebensqualität. Die Schaffung neuer, anspruchsvoller Arbeitsplätze ist Teil der Wirkung eines Zentrums. Unternehmen im TGZ sollten vorzugsweise Neugründungen sein, die aus der Hochschule und Forschungsinstituten oder aus größeren, am Markt erfahrenen Unternehmen entstehen. Studentische Initiativen und solche von Absolventen sind besonders zu wecken und zu fördern. Hierin sieht das TGZ einen wichtigen Schwerpunkt seiner Tätigkeit.

Nach Einschätzung einer vom BMBF 1998 vorgelegten Studie (6) zeichnet sich in Deutschland eine neue Gründungswelle ab. Dabei haben technologieorientierte Unternehmensgründungen seit 1990 eine starke Dynamik erlebt. Es wird eingeschätzt, dass das in Hochschulen, außeruniversitären Forschungseinrichtungen und in F\&E-Abteilungen von Unternehmen schlummernde Potenzial bei weitem noch nicht ausgeschöpft ist. Diese mögliche Gründungswelle muß sowohl durch das TGZ als auch durch die TFH durch vielfältige Aktivitäten gefördert werden.

An der Wildauer Hochschule hat der Gründungsgedanke bereits einen festen Platz in diversen Lehrangeboten gefunden. Im Studienschwerpunkt Unternehmensfuihrung und in der Logistik gibt es Pflichtveranstaltungen zur Unternehmensgründung/-sanierung und im Bereich Betriebswirtschaft wird Unternehmensgründung als Wahlpflichtfach angeboten.

Die Zahl studentischer Gründungen, die während oder nach dem Studium stattfinden, ist nicht exakt feststellbar. Es gibt weder flächendeckende Erhebungen noch eine Meldepflicht und kaum Möglichkeiten, den Weg einzelner Studenten nach Verlassen der Hochschule zu verfolgen. Derzeit sind etwas mehr als 20 studentische Gründungen aus der Wildauer Hochschule bekannt. Sie haben sich u.a. in Gründerseminaren dargestellt. Diese Zahl ist für eine kleine und relativ junge Hochschule recht beachtlich (7). Trotzdem muß ganz besonders vonTGZ und TFH, Hochschullehrern und Unternehmern überlegt werden, wie eine zunehmende Zahl von Studenten und Absolventen motiviert und befähigt werden kann, eine eigene selbständige, tragfähige Existenz aufzubauen.
Spricht man von Grïndungen aus der Hochschule, meint man in aller Regel studentische Gründungen. Das reicht aber nicht aus. Gründungen wissenschaftlicher Mitarbeiter sind ebenso einzubeziehen, wie Gründungen von Hochschullehrern.

Auch diese Gruppen werden bei den Bemühungen um Existenzgründungen aus TFH Wildau berücksichtigt, und Ansiedlungen im Wildauer TGZ sind in Aussicht.

Die Gründung neuer Unternehmen zur Umsetzung von Logistik-Innovationen in Zusammenarbeit mit der TFH Wildau erscheint insbesondere auf den Gebieten virtueller Produktionslösungen, Tele-Service, Verkehrsoptimierung, Entsorgung durch intelligente Containerlösungen in der Kreislaufwirtschaft, Software für Animation und Simulation oder im Bereich des Luftverkehrs, insbesondere bei der Gepäckabfertigung, möglich.

Die Stimulierung von Gründeraktivitäten erscheint durch eine Wildauer Existenzgründer Werkstatt, für die die 1995 initiierte Innovationswerkstatt Wildau als Kommunikationsplattform genutzt werden könnte, verbunden mit der Auslobung eines Wildauer ExistenzGründerpreises angebracht.

Dabei wären die hohe fachliche, wirtschaftliche und gesellschaftliche Kompetenz von Hochschule, Unternehmen und TGZ, insbesondere unter Einbeziehung seines Beirats, zusammenzuführen. Vorreiter und nachahmenswerte Beispiele renommierter Institutionen hat es in letzter Zeit reichlich gegeben (8). Doch reichen sie allein nicht aus, um der Existenzgründungsbewegung den notwendigen Schwung zu geben. Wichtig erscheinen uns die Umsetzung der bundesweiten Erfahrungen auf lokaler Ebene.

Das hier Vorgestellte ist in wesentlichen Teilen noch Vision, bisher nur in Ansätzen vorhanden. Die angestrebte Profilierung erscheint sinnvoll und machbar. Darüber hinaus sind im TGZ Wildau Existenzgründer, Jungunternehmer oder Ausgründer aller Branchen willkommen, deren Geschäftsfelder innovative, technologieorientierte Ansprüche erfuillen, zugleich über ein solides kaufmännisches Konzept verfügen. 


\section{Literatur}

[1] Dr. E. Brink, Das Technologie- und Gründerzentrum Wildau - eine Zwischenbilanz, Wiss. Beiträge der TFH Wildau, 1/1997, 6-10

Dr. E. Brink Tradition \& Moderne - Die Gemeinde Wildau und das TGZ Wildau

Technik \& Gesellschaft - Forum des VDI Berlin-Brandenburg, 3/1999, 28-29

[2] Dr. G. Kayser - Der Gründerberater Nr.7/1999

[3] Dr. E. Brink - Regionalwettbewerb der Technologie- und Gründerzentren im Land Brandenburg - Ideenskizze TGZ Wildau, 22.10.1998

[4] Dr. B. Hentschel - Innovative Grenzlogistik - Beiträge zur 4. Polnisch-Deutschen Logistikkonferenz, 01.07.99 in Slubice, in der Wildauer Schriftenreihe Logistik, Bd.2, 1999

[5] Studienfuihrer 1999/2000, TFH Wildau

[6] ADT - Arbeitsgemeinschaft Deutscher technologie- und Gründerzentren - Projekt ATHENE, Mai 1998

[7] Dr. Ing. H. Stoltenberg - Hochschulen als Unternehmensgründer, markt und mark 06/1999

[8] Business Plan Wettbewerb '98 Berlin-Brandenburg

Kienbaum Dienstleistungspreis - Förderpreis für zukunftsorientierte Dienstleistungen

Start Up Wettbewerb der Sparkassen

Berlin-Brandenburger Innovations- und Gründungsoffensive (BINGO)

Gründerwettbewerb Multimedia 1999 (BMWi)

Gründen, Festigen, Wachsen - Ein Leitfaden für Unternehmerinnen und Unternehmer im Land Brandenburg

GründungsDialog - Förderung und Unterstützung von technologieorientierten Unternehmensgründungen in den neuen Bundesländern und Berlin (Ost) (bmb+f)

\section{Autoren}

Dr. rer. nat. Eberhard Brink

Geschäftsführer der Technologie- und Gründerzentrum Wildau $\mathrm{GmbH}$

Tel. (0 33 75) 50294

Fax (0 33 75) 508-289

E-Mail: tgz@tf.tfh-wildau.de

\section{Prof. Dr.-Ing. habil. Bernd Hentschel}

Unternehmenslogistik/Produktionslogistik

Tel. (0 33 75) 508-927

Fax (0 33 75) 508-928

E-Mail: bhentsch@wi-bw.tfh-wildau.de

Dr. Ing. Hartmut Stoltenberg

Leiter der Technologie- und Innovationsberatungsstelle der TFH Wildau

Tel. (0 33 75) 508-125

Fax (0 33 75) 508-213

E-Mail: stoltenb@tf.tfh-wildau.de 